\title{
Cross-network interaction for diagnosis of major depressive disorder based on resting state functional connectivity
}

\author{
Xueling Zhu ${ }^{1,2} \cdot$ Fulai Yuan $^{3} \cdot$ Gaofeng Zhou ${ }^{1} \cdot$ Jilin Nie ${ }^{1} \cdot$ Dongcui Wang ${ }^{1} \cdot$ Ping Hu$^{1} \cdot$ Lirong Ouyang $^{1}$. \\ Lingyu Kong ${ }^{1} \cdot$ Weihua Liao ${ }^{1,2}$
}

Published online: 30 July 2020

(C) The Author(s) 2020

\begin{abstract}
Previous studies have suggested that resting-state functional connectivity plays a central role in the physiopathology of major depressive disorder (MDD). However, the individualized diagnosis of MDD based on resting-state functional connectivity is still unclear, especially in first episode drug-naive patients with MDD. Resting state functional magnetic resonance imaging was enrolled from 30 first episode drug-naive patients with MDD and age- and gender-matched 31 healthy controls. Whole brain functional connectivity was computed and viewed as classification features. Multivariate pattern analysis (MVPA) was performed to discriminate patients with MDD from controls. The experimental results exhibited a correct classification rate of $82.25 \%(p<0.001)$ with sensitivity of $83.87 \%$ and specificity of $80.64 \%$. Almost all of the consensus connections $(125 / 128)$ were cross-network interaction among default mode network (DMN), salience network (SN), central executive network (CEN), visual cortex network (VN), Cerebellum and Other. Moreover, the supramarginal gyrus exhibited high discriminative power in classification. Our findings suggested cross-network interaction can be used as an effective biomarker for MDD clinical diagnosis, which may reveal the potential pathological mechanism for major depression. The current study further confirmed reliable application of MVPA in discriminating MDD patients from healthy controls.
\end{abstract}

Keywords Major depressive disorder - Multivariate pattern analysis - Resting-state fMRI $\cdot$ Functional connectivity · Cross-network interaction

\section{Introduction}

As one of the most common psychiatric disorders worldwide, major depressive disorder (MDD) is characterized by persistent, pervasive feelings of sadness, guilt, and worthlessness, which leads to serious economic impact to the families and bring great burden to the society (World Health Organization 2017). However, the diagnosis of MDD is still challenging because the diagnosis is primarily based on both the patient's

Weihua Liao

109731926@qq.com

1 Department of Radiology, Xiangya Hospital, Central South University, Changsha, China

2 National Clinical Research Center for Geriatric Disorders, Xiangya Hospital, Central South University, Changsha, China

3 Health Management Center, Xiangya Hospital, Central South University, Changsha, China self-reported symptoms and the psychiatrist's experience (American Psychiatric Association 2013). Reliable MDD detection becomes difficult especially in the condition without experienced psychiatrist, which limits subsequent treatment of this disease (Mitchell et al. 2009; Nabbe et al. 2017). Obviously, it is necessary to develop an automated and objective method to help to diagnosis MDD.

In the last decade, multi-modal magnetic resonance imaging (MRI) techniques have been widely used to characterize the underlying pathophysiology of mental diseases (Buckner 2010). Compared with the task MRI, resting-state fMRI has attracted considerable attention owing to easier implementation and fewer requirements to the patients (Greicius et al. 2003; Fox and Raichle 2007). Although some studies have focused on investigating dynamic functional connectivity ( $\mathrm{Li}$ et al. 2019; Liao et al. 2019) or combined dynamic and static connectivity (Liao et al. 2018), resting-state functional connectivity (static) has always been proven effective in revealing the alterations of brain functional networks in neuropsychiatric disorders including Alzheimer (Badhwar et al. 2017), 
schizophrenia (Dong et al. 2018) and depression (Greicius et al. 2007; Greicius 2008).

The default mode network (DMN) refers to some brain areas that form an integrated system for self-related activity, including autobiographical, self-monitoring and social functions, which mainly contained the medial prefrontal cortex, anterior cingulate cortex, posterior cingulate cortex, inferior parietal lobule, parahippocampal gyrus and hippocampus (Raichle et al. 2001). The salience network (SN) usually is involved in processing emotion or monitoring for salient events (Seeley et al. 2007), which included insula, amygdala, temporal poles, super temporal gyrus, pallidum and caudate (Menon 2011). The central executive network (CEN) is responsible for high-level cognitive functions, notably the control of attention and working memory (Mulders et al. 2015), which were anchored in dorsolateral prefrontal cortex, and posterior parietal cortex (Seeley et al. 2007; Habas et al. 2009). Although findings are somewhat inconsistent, previous studies have revealed that the pathophysiology of MDD involves a large-scale dysfunction in brain functional networks such as DMN, SN and CEN (Greicius et al. 2007; Sexton et al. 2012; Zhu et al. 2012; Hamilton et al. 2013; Guo et al. 2014b; Manoliu et al. 2014a). However, most of these studies traditionally adopt the univariate analysis, which has neglect the highly interconnected nature of the brain (Davatzikos, 2004). Whether altered resting-state functional connectivity could be used in the individualized diagnosis of MDD is still unknown.

How to differentiate MDD at the individual level is the key problem to be settled. With the incoming of artificial intelligence era, machine learning methods have been used widely in brain image analysis (Liu et al. 2012; Smith 2012; Liu et al. 2015).As one of the typical supervised machine learning methods, multivariate pattern analysis (MVPA) can extract stable identification features from brain image data to differentiate patients from healthy controls at the individual subject level (Orru et al. 2012; Wolfers et al. 2015). In contrast to the univariate statistical methods, MVPA could further detect exciting spatially distributed information to highlight neural mechanisms of psychiatric disease. An increasing number of neuroimaging studies focused on applying MVPA to discriminate MDD patients from healthy controls (Fu et al. 2008; Craddock et al. 2009; Liu et al. 2012; Zeng et al. 2012; Ma et al. 2013; Zhong et al. 2017). A recent meta-analysis of multivariate pattern recognition studies to differentiate patients diagnosed with MDD from healthy controls has confirmed high representational capacity of MVPA methods to identify neuroimaging-based biomarkers of depression (Kambeitz et al. 2017). It is noteworthy that resting state functional connectivity has been proved to be superior classification accuracy of diagnostic models, compared with structural MRI or task-based fMRI data (Kambeitz et al. 2017).

Several studies have demonstrated the clinical value of resting state functional connectivity to distinguish MDD from healthy controls based on MVPA methods (Zeng et al. 2012; Zhong et al. 2017). The majority of the most discriminating functional connections were located within or across different resting state networks, such as DMN, SN and CEN, which were related to emotional and cognitive function (Zeng et al. 2012; Ma et al. 2013). In addition to methodological difference among these studies, the variable diagnostic performance may due to demographic and clinical characteristics of depressed patients. It was reported that antidepressant medicine and old age could cause alterations in brain function and structures (Anand et al. 2005; Guo et al. 2014c). Therefore, it was necessary and crucial to explore neuroimaging-based diagnostic models in first episode treatment-naïve young major depression. Though a few pioneering studies have now emerged on these topics, no final conclusion has yet been reached (Guo et al. 2014a; Zheng et al. 2019).

The aim of this study was to explore diagnostic models at an individual level to differentiate patients with MDD from healthy controls. MVPA and resting state functional connectivity were used as a diagnostic tool in first-episode, treatmentnaive young adults with MDD and carefully matched healthy control subjects. We hypothesized that relative to healthy controls, abnormal cross-network functional connectivity were expected to be observed in resting state networks involved in emotional and cognitive function in MDD group.

\section{Materials and methods}

\section{Participants}

Patients with MDD were recruited from the psychiatric clinic at Xiangya Hospital of Central South University in Changsha, China. Patients with MDD were diagnosed according to the Structured Clinical Interview for DSM-IV by independent assessments of two psychiatrists. All of the patients were experiencing their first episode of depression and had never received medication. Closely matched healthy subjects were recruited through advertisements from several colleges in Changsha. All subjects were right-handed. In order to reduce the influence of addictive substance, all subjects were required to be abstinent from caffeine, nicotine, alcohol and other addictive substance at least one week prior to the fMRI scanning. The shared exclusion criteria for patients and control subjects included any major medical illnesses; clinical diagnosis of neurologic trauma; any history of psychiatric disorder in the control subjects or any history of psychiatric disorder, except major depression, in the MDD patients; any history of substance abuse or alcohol in the past 6 months; and any contraindications to imaging scanning. Finally, 30 patients with MDD and 31 matched healthy controls were recruited (Table 1).

Written informed consent was obtained from all participants prior to the study, which was approved by the Institutional 
Table 1 Demographic and clinical characteristics of the MDD and control groups

\begin{tabular}{lllrll}
\hline Characteristic & MDD & Control & $\mathrm{t} / \chi^{2}$ & $\mathrm{p}$ & Cohen's d \\
\hline Age & $22.29 \pm 1.47$ & $21.18 \pm 3.32$ & 0.43 & 0.72 & 0.15 \\
Sex (female/male) & $16 / 14$ & $16 / 15$ & 0.01 & 0.84 & \\
CES-D & $39.25 \pm 5.75$ & $18.33 \pm 5.14$ & 13.10 & 0 & 3.12 \\
Age at onset (years) & $21.17 \pm 3.22$ & NA & & & \\
Illness duration (months) & $8.35 \pm 4.46$ & NA & & & \\
\hline
\end{tabular}

Review Board of Xiangya Hospital of Central South University for Brain Research. The methods were conducted in accordance with relevant approved guidelines and regulations.

\section{Measures}

Depressive severity was measured using the CES-D scale (Radloff 1977), a 20-item self-report instrument to assess depressive symptoms in the general population. The Chinese version of the CES-D has been found to have high degrees of reliability and validity (Wang et al. 2013). In this study, the internal consistency of the CES-D was good (Cronbach's alpha $=0.93)$.

\section{MRI data acquisition}

Resting state fMRI images were captured by a 3T Siemens Magnetom Symphony scanner. During scanning, all participants were asked to rest with their eyes closed and to try not to think of anything systematically. All subjects placed their heads in a standard head coil (16-channel). Participants were positioned comfortably on the scanner bed and fitted with soft ear plugs; foam pad was used to minimize head movement.

Functional images were obtained axially using a singleshot, gradient-recalled echo-planar imaging sequence parallel to the line of the anterior-posterior commissure: repetition time $/$ echo time $=2000 / 40 \mathrm{~ms}$, thickness $/ \mathrm{gap}=5 / 0 \mathrm{~mm}$, field of view $=240 \times 240 \mathrm{~mm}$, flip angle $=90^{\circ}$, matrix $=64 \times 64$, slices $=26,150$ volumes.

High-resolution T1-weighted images were also acquired with a three-dimensional spoiled gradient-recalled sequence in an axial orientation: repetition time $=8.5 \mathrm{msec}$, echo time $=$ $3.2 \mathrm{~ms}$, flip angle $=15^{\circ}$, field of view $=25 \mathrm{~cm}$, matrix $=$ $256 \times 256$, slice thickness $=1.0 \mathrm{~mm}, 176$ slices.

\section{Image preprocessing}

Image preprocessing was carried out using the Data Processing \& Analysis for Brain Imaging software package (DPABI, http:// rfmri.org/dpabi). After discarding of the first 10 volumes of each functional time series, slice timing, and realignment of head motion, data from three patients and two healthy subjects were excluded because their translation or rotation exceeded $\pm 1.5 \mathrm{~mm}$ or $\pm 1.5^{\circ}$. The images were then spatially normalized to a standard template (Montreal Neurological Institute, Montreal, Quebec, Canada). The sources of spurious variance were regressed out including 6 parameters from head-motion correction (Friston 24-parameter model), white matter and cerebrospinal fluid signal. The resulting images were spatially smoothed with a Gaussian filter of $8 \mathrm{~mm}$ full-width half-maximum kernel. Linear detrending and temporal bandpass $(0.01-0.08 \mathrm{~Hz})$ filtering were performed to remove low-frequency drifts and physiological high-frequency noise. In view of the influence of head motion on functional connectivity results, the data was further performed with the scrubbing method to remove time points affected by head motions (Yan et al. 2013; Power et al. 2014).

\section{Anatomical parcellation}

The registered functional MRI volumes with the Montreal Neurological Institute template were divided into 116 regions according to the automated anatomical labelling atlas (Tzourio-Mazoyer et al. 2002). The atlas divides the cerebrum into 90 regions (45 in each hemisphere) and divides the cerebellum into 26 regions ( 9 in each cerebellar hemisphere and 8 in the vermis). We evaluated functional connectivity between pairs of regions by calculating Pearson correlation coefficients. For each subject, we obtained a resting-state functional network captured by a $116 \times 116$ symmetric matrix. According to previous studies (Menon 2011, 2018; Supekar et al. 2019), these regions were divided into six different resting-state networks, such as DMN, SN, CEN, VN, Cerebellum and Other. The VN mainly comprised lingual gyrus, fusiform, cuneus and occipital lobe (Zeng et al. 2012).

\section{MVPA}

\section{Feature selection}

Feature selection was used to construct the feature space for classification by retaining the most discriminating functional connections. The discriminative power of a feature can be quantitatively measured. The F score method was used for feature ranking in this current study for its simplity and effectiveness (Chen and Lin 2006), which has been widely used in mental disease (Liu et al. 2015; Chen et al. 2016) and other fields (Wang 2007; Akay 2009). $F$ score of the $i$ th feature is defined as follows: 


$$
F(i)=\frac{\left(\bar{x}_{i}^{(+)}-\bar{x}_{i}\right)^{2}+\left(\bar{x}_{i}^{(-)}-\bar{x}_{i}\right)^{2}}{\frac{1}{n_{+}-1} \sum_{k=1}^{n_{+}}\left(\bar{x}_{k, i}^{(+)}-\bar{x}_{i}^{(+)}\right)^{2}+\frac{1}{n_{-}-1} \sum_{k=1}^{n_{-}}\left(\bar{x}_{k, i}^{(-)}-\bar{x}_{i}^{(-)}\right)^{2}}
$$

where $\bar{x}_{i}$ is the average of the $i$ th feature of the whole data set, $\bar{x}_{i}^{(+)}$and $\bar{x}_{i}^{(-)}$are the averages of the $i$ th feature of MDD and healthy control data sets; $x_{k, i}^{(+)}$is the $i$ th feature of the $k$ th MDD instance, and $x_{k, i}^{(-)}$is the $i$ th feature of the $k$ th healthy control instance. The denominator represents the discrimination within each of the MDD and healthy control sets, while the numerator represents the discrimination between the two sets. Obviously, the larger the $F$ score is, the more likely the feature is of more potential to discriminate the groups.

\section{Support vector machine classification}

Support vector machine (SVM) classifier was adopted for classification, which works well when the number of training samples is small but the number of features is large (Vapnik 2000). SVM classification is one type of supervised learning which consists of two steps: training and testing. During the training step, SVM forms the decision function from the training data set with its class labels. During the testing step, it predicts the class labels of new test examples (Liu et al. 2015). A linear kernel SVM was used, in order to reduce the risk of overfitting the data and allow direct extraction of the feature weights (Pereira et al. 2009). The SVM classifier was implemented using LIBSVM toolbox with default parameters (Chang and Lin 2011).

\section{Evaluation of the performance of the classifier}

Due to our limited number of samples, a LOOCV strategy was employed to evaluate the performance of the classifier (Scholkopf and Smola 2001; Liu et al. 2015; Chen et al. 2016). In brief, suppose there were $n$ samples in total. In each LOOCV trial, $n-1$ samples were used as the training set and the remaining one was used as the testing set. This procedure was repeated $n$ trials. Classifiers were built for each training set and tested with its corresponding testing subject. Accuracy, sensitivity, and specificity could be used to quantify the performance of the classifier based on the results of LOOCV. Utilizing of LOOCV strategy could get stable weights of each feature and the weights got from the training dataset were more close to the whole dataset (Anderson et al. 2014).

\section{Classification weight definition}

In each trial of LOOCV, the final features used in classification differed because feature ranking was based on a slightly different subset of the data. Consensus features were defined (Liu et al. 2015; Chen et al. 2016). They were regarded as the common features always selected to form the final features set from each LOOCV iteration. The weight of the consensus feature was the average value of the classification weight across all trials of LOOCV. The weight of a consensus feature was defined as zero if this connection was not selected as a classification feature.

To represent the relative contribution of different regions for classification, the classification weight of each region was evaluated by summing one-half of the classification weight of the connections associated with that region (splitting the weight of connections into the regions they connects) (Meier et al. 2012). Of note, if a region did not form any consensus feature, it was given a region weight of zero. We defined a region (consensus feature) with greater weights if its weight was at least 1 standard deviation greater than the average of the weight of all the regions (consensus features) (Tian et al. 2011; Liu et al. 2015).

\section{Permutation test of classification performance}

To estimate the statistical significance of observed classification accuracy is a challenging problem due to the high dimensionality of the fMRI data and the relatively small number of training examples. Some researchers have proposed a framework of permutation test, which is a nonparametric technique in which a reference distribution is obtained by calculating all possible values of the test statistic under rearrangements of the labels of the samples (Golland and Fischl 2003). The permutation test has widely used in classifying brain states (Mourao-Miranda et al. 2005), sexual dimorphism (Wang et al. 2012) and resting-state brain function (Zhu et al. 2008; Liu et al. 2015). In the current analysis, the class labels of the training data were randomly permuted 1000 times. The same entire classification process including feature selection was carried out with each set of permuted class labels. The accuracies were obtained across all permutations. Based on these null probability distributions and the observed statistic corresponding to the actual labeling, $p$ value was calculated as the proportion of accuracies that are equal to or greater than the accuracy obtained by the non-permutated (original) data (Liu et al. 2015). The smaller is the $p$ value, the more reasonable to reject the null hypothesis. Usually a threshold of $p<0.05$ is meaningful.

\section{Result}

A relatively high classification accuracy of $82.25 \%$ was achieved in this study (sensitivity $83.87 \%$, specificity $80.64 \%, p<0.001)$. The receiver operating characteristic (ROC) curve of the classifier was shown in Fig. 1. The area under the ROC curve (AUC) was 0.892, indicating a good classification power. 
Fig. 1 ROC curve of the classifier

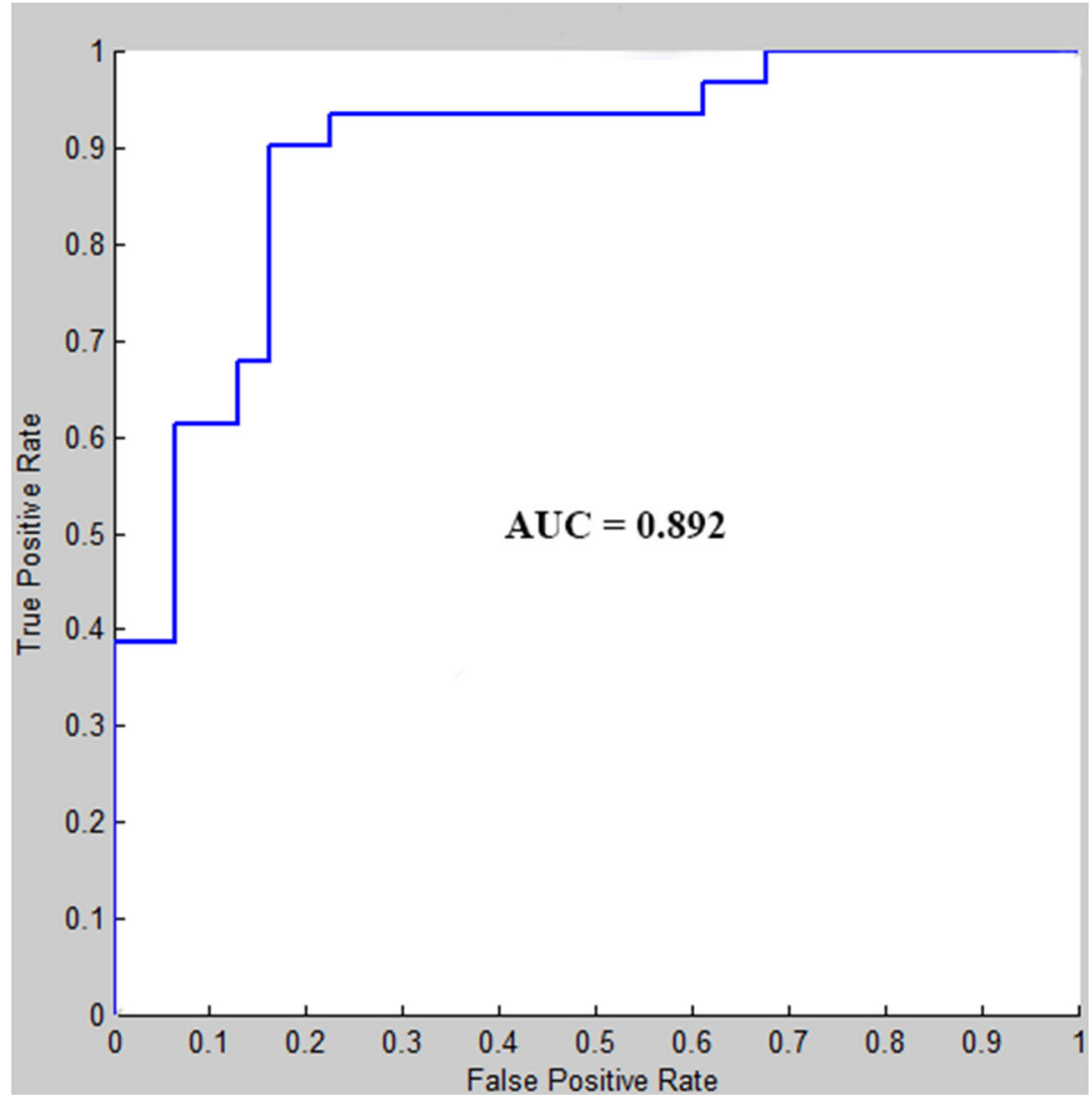

128 consensus features were identified in the cross-validation. Similar to the previous studies, brain regions related to consensus functional connectivity were found to be located primarily in 6 resting state networks: DMN, SN, CEN, VN, Cerebellum and Other. Almost the entire consensus connections $(125 / 128)$ used to distinguish MDD from healthy controls belonged to the cross-network interaction. Some consensus features exhibited greater weights than others, which means that its weight was at least one standard deviation greater than average weight of all the regions (Tian et al. 2011). 128 consensus features were shown in Fig. 2.

The mean weight of consensus features across two networks is one indicator to represent the role of interactions across these two networks to some extent. Therefore, the mean weight of consensus features cross 6 networks was calculated. Crossnetwork interactions in 6 networks were constructed in Fig. 3. Some cross-network interactions exhibited greater weights than others. The cross-network interactions with greater weight were mainly located across DMN, SN, CEN and VN.

Several brain regions exhibited greater weights than others. These regions contain right supramarginal gyrus and right infer parietal lobule (involved in DMN), super temporal gyrus and left putamen (involved in SN), left super occipital gyrus, and lingual gyrus (involved in $\mathrm{VN}$ ). Of all, the supramarginal gyrus exhibited highest discriminative power. Figure 4 showed these regions.

\section{Discussion}

In this study, resting state functional connectivity was used as the feature to identify first-episode, drug-naïve MDD patients from health populations using MVPA methods. Four main results were revealed: (1) a correct classification rate was $82.25 \%$ and the AUC value was 0.892 , indicating the important value of whole brain resting state functional connectivity to identify MDD patients from healthy controls; (2) almost all of the consensus connections $(125 / 128)$ used to distinguish MDD belonged to cross-network connection among DMN, SN, CEN, VN, Cerebellum and Other; (3) The consensus connections with greater weight were mainly located across DMN, SN, CEN and VN. (4) The supramarginal gyrus exhibited the highest discriminative power.

Consistent with previous findings, cross-network interaction was found to be altered in patients with MDD. DMN, $\mathrm{SN}, \mathrm{CEN}, \mathrm{VN}$ and Cerebellum have been commonly regarded as key resting state networks in MDD, with abnormalities having been observed in blood oxygenation level-dependent fMRI activation, as well as in baseline metabolism or perfusion (Gong and He 2015; Mulders et al. 2015). However, it should be noted that the networks did not function independently. It may be not enough to only investigate the connectivity within one specific network. In addition to the within-network connectivity, cross-network 


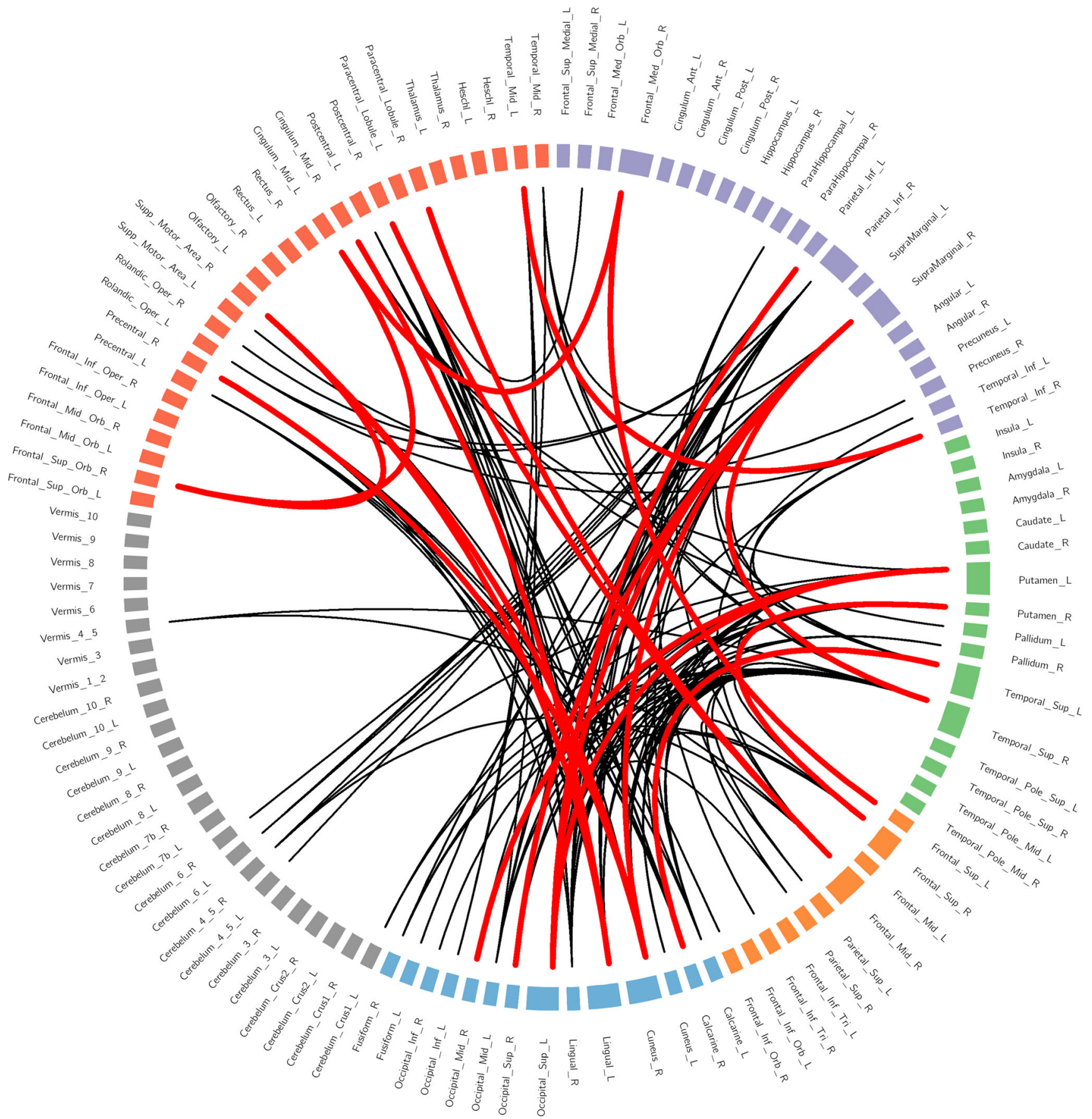

Fig. 2 Regions and distribution of 128 consensus features. Different colors of nodes belong to different networks. Purple means default mode network (DMN), green means salience network (SN), orange means central executive network (CEN), blue means visual cortical network (VN), grey means Cerebellum network, red means Other

connectivity was also investigated by a large number of studies on resting-state functional connectivity in MDD (Brakowski et al. 2017). The interplay between the DMN and CEN and their sub-networks have been hot topics in this field (Mantini et al. 2007; Manoliu et al. 2014b; Zhu et al. 2017; Liu et al. 2018). A meta-analysis study network. Red lines represent the connections with more classification weight, and black lines represent the connections whose classification weight were under the mean plus/minus the standard error of all connections used as classification features

suggested that altered connectivity between neural systems involved in cognitive control and those that support salience or emotion processing may relate to deficits regulating mood in MDD (Kaiser et al. 2015). Our study extends these prior findings by providing new evidence for abnormal resting-state functional connectivity in MDD. 
Fig. 3 Cross-network interaction among 6 resting state networks. Red lines represent connections with greater weight than mean weight plus the standard error of all connections; black lines represent left connection except the greater connections

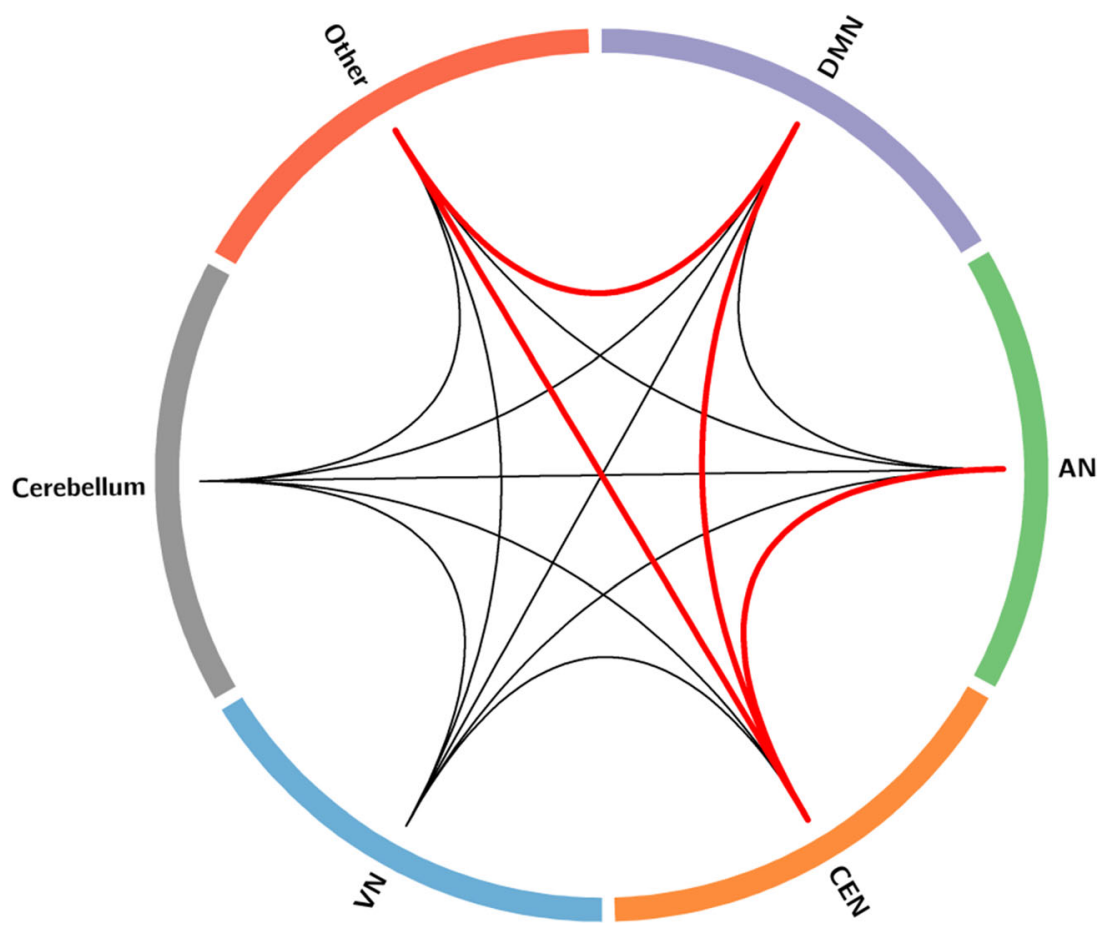

It is worth noting that aberrant functional organization of DMN, SN and CEN was observed in the resting-state data of MDD subjects. The recently proposed "triple-network" model emphasized the corporation among these three networks, which has been revealed to underlie a wide range of psychopathologies, including schizophrenia, autism and attentiondeficit/hyperactivity disorder (Bressler and Menon 2010;
Menon 2011). Dysfunction of three networks has remarkably occurred in many mental and neurological disorders (Manoliu et al. 2014b). With a similar pathophysiological mechanism observed in schizophrenia, MDD is reported to demonstrate common causal dysconnectivity between DMN and SN, as well as opposing functional dysconnectivity of DMN-CEN and SN-CEN (Jiang et al. 2017). Significantly decreased
Fig. 4 Nodes with greater classification weight which is higher the mean plus the standard error of all connections. Nodes with purple color were located in $\mathrm{DMN}$, green in $\mathrm{SN}$, orange in $\mathrm{CEN}$, blue in $\mathrm{VN}$, grey in Cerebellum, and red means Other network

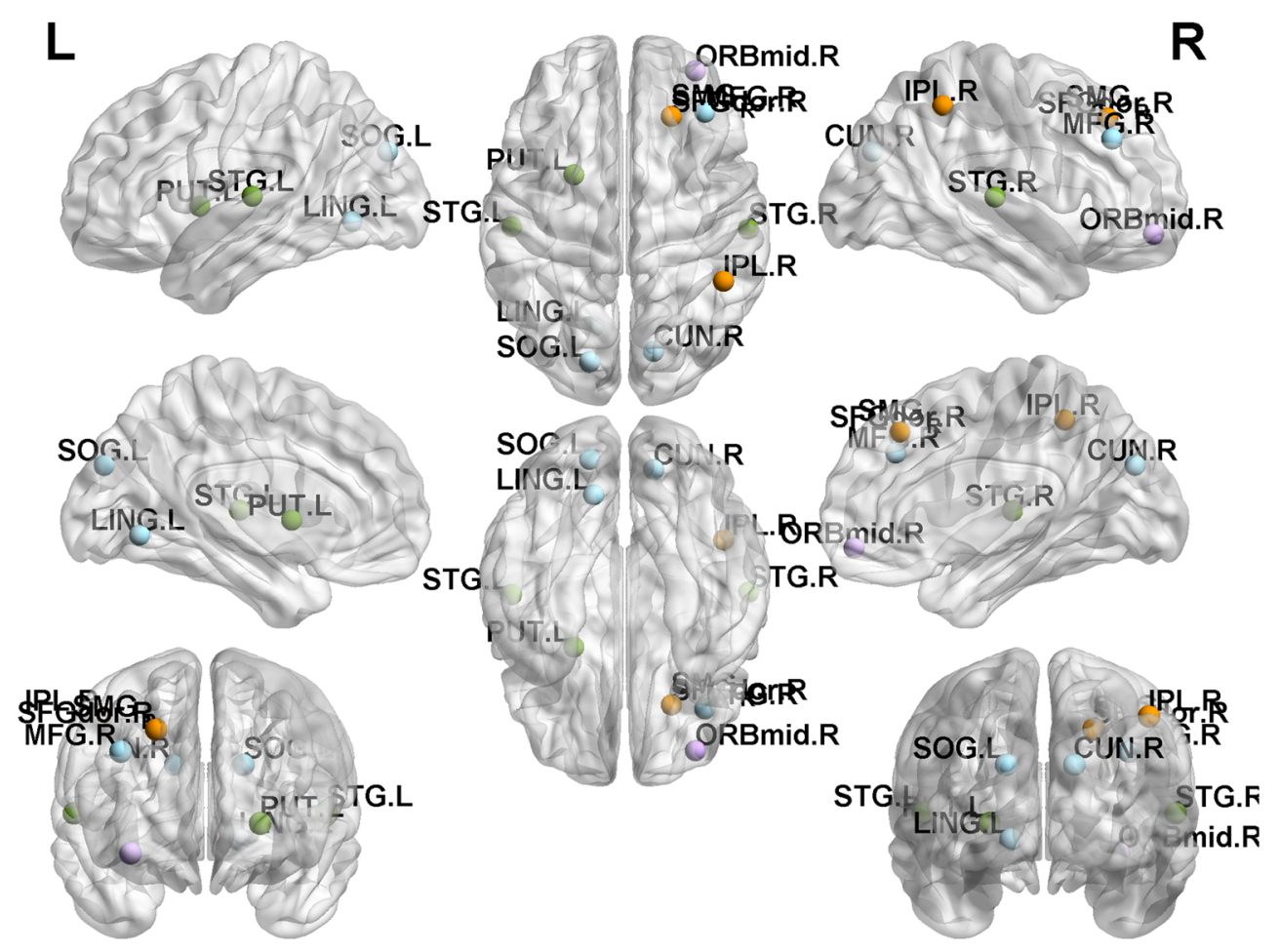


interaction degree between DMN and CEN was reported in MDD (Zheng et al. 2015). Consistent with previous studies, our study suggested that the abnormal triple networks interaction in resting state of MDD patients.

As a part of DMN, the supramarginal gyrus showed highest classification weight in present study. Great difference in the functional connectivity of the DMN between individuals with MDD and healthy controls has been revealed (Greicius et al. 2007; Zhu et al. 2012; Hamilton et al. 2015). As anterior to the junction of parietal and temporal cortex, the supramarginal gyrus is traditionally known to be involved in several cognitive functions, including speech repetition, auditory shortterm memory (Buchsbaum and D'Esposito 2009; Baldo et al. 2012). However, recent converging evidence from multiple methods and experiments that the supramarginal gyrus is crucial for overcoming emotional egocentricity in social judgement, which is closely associated with self-referential processing (Silani et al. 2013). Damage of supramarginal gyrus and adjacent areas can produce a variety of disorders associated with distorted body knowledge and self-awareness (Berlucchi and Aglioti 1997).

Although the classification accuracy of this present study was favorable, several limitations should be noted. As well as many previous studies in this field (Zeng et al. 2012; Liu et al. 2015; Zhong et al. 2017), the first limitation is related to small sample size with no comorbid conditions, so we may be cautious in generalizing the findings of this study to more larger samples with comorbid diagnoses. In the future, larger sample size, multicenter imaging data and a large independent test data set are welcome to confirm the classification results. Secondly, we only explored resting state functional connectivity and did not consider brain structural connectivity, dynamic functional connectivity (Li et al. 2019; Liao et al. 2019) or combined dynamic and static connectivity (Liao et al. 2018). Functional and structural, static or dynamic imaging data will be combined to provide more reliable diagnostic information. Thirdly, automated anatomical labeling atlas was used in this study. Previous studies revealed that different templates could impact the generated connections at a certain degree, more brain templates would be used to confirm the accuracy.

\section{Conclusion}

In summary, we have demonstrated multivariate pattern analysis methods can identify first episode drug-naive patients with MDD from healthy controls based on resting-state functional connectivity with a correct classification rate of $82.25 \%$ $(p<0.001$, sensitivity $83.87 \%$, specificity $80.64 \%)$. Almost all of the most discriminating consensus connections were cross-network connectivity among DMN, SN, CEN, VN, Cerebellum and Other network, which implied the emotional and cognitive impairments characteristic of MDD. Moreover, the supramarginal gyrus located in DMN exhibited the highest discriminative power in classification. The current study further confirmed reliable application of MVPA in the discriminating MDD patients from healthy controls. More importantly, these results support the cross-network interaction as an effective biomarker for MDD clinical diagnosis, which may reveal the potential pathological mechanism for major depression.

Acknowledgements This research was supported by grants from the National Natural Science Foundation of China (61972460 and 61802443), Hunan Province Science Fund for Distinguished Young Scholars (2019JJ20037), Foundation for the Author of National Excellent Doctoral Dissertation of PR China (201411), Philosophy and Social Science Fund Project of Hunan Province (17YBA426), Youth Science fund of Xiangya hospital, central south university (2017Q19).

\section{Compliance with ethical standards}

Conflict of interest The authors declare no conflict of interest.

Open Access This article is licensed under a Creative Commons Attribution 4.0 International License, which permits use, sharing, adaptation, distribution and reproduction in any medium or format, as long as you give appropriate credit to the original author(s) and the source, provide a link to the Creative Commons licence, and indicate if changes were made. The images or other third party material in this article are included in the article's Creative Commons licence, unless indicated otherwise in a credit line to the material. If material is not included in the article's Creative Commons licence and your intended use is not permitted by statutory regulation or exceeds the permitted use, you will need to obtain permission directly from the copyright holder. To view a copy of this licence, visit http://creativecommons.org/licenses/by/4.0/.

\section{References}

Akay, M. F. (2009). Support vector machines combined with feature selection for breast cancer diagnosis. Expert Systems with Applications, 36(2), 3240-3247. https://doi.org/10.1016/j.eswa. 2008.01.009.

American Psychiatric Association. (2013). Major depressive disorder, diagnostic and statistical manual of mental disorders 5. Washington, DC: American Psychiatric Association.

Anand, A., Li, Y., Wang, Y., Wu, J. W., Gao, S. J., Bukhari, L., et al. (2005). Antidepressant effect on connectivity of the moodregulating circuit: An fMRI study. Neuropsychopharmacology, 30(7), 1334-1344. https://doi.org/10.1038/sj.npp.1300725.

Anderson, A., Douglas, P. K., Kerr, W. T., Haynes, V. S., Yuille, A. L., Xie, J., Wu, Y. N., Brown, J. A., \& Cohen, M. S. (2014). Nonnegative matrix factorization of multimodal MRI, fMRI and phenotypic data reveals differential changes in default mode subnetworks in ADHD. Neuroimage, 102(Pt 1), 207-219. https://doi.org/10. 1016/j.neuroimage.2013.12.015.

Badhwar, A., Tam, A., Dansereau, C., Orban, P., Hoffstaedter, F., \& Bellec, P. (2017). Resting-state network dysfunction in Alzheimer's disease: A systematic review and meta-analysis. Alzheimers Dement (Amst), 8, 73-85. https://doi.org/10.1016/j. dadm.2017.03.007. 
Baldo, J. V., Katseff, S., \& Dronkers, N. F. (2012). Brain regions underlying repetition and auditory-verbal short-term memory deficits in aphasia: Evidence from voxel-based lesion symptom mapping. Aphasiology, 26(3-4), 338-354. https://doi.org/10.1080/02687038. 2011.602391.

Berlucchi, G., \& Aglioti, S. (1997). The body in the brain: Neural bases of corporeal awareness. Trends in Neurosciences, 20(12), 560-564.

Brakowski, J., Spinelli, S., Dorig, N., Bosch, O. G., Manoliu, A., Holtforth, M. G., et al. (2017). Resting state brain network function in major depression - depression symptomatology, antidepressant treatment effects, future research. Journal of Psychiatric Research, 92, 147-159. https://doi.org/10.1016/j.jpsychires.2017.04.007.

Bressler, S. L., \& Menon, V. (2010). Large-scale brain networks in cognition: Emerging methods and principles. Trends in Cognitive Sciences, 14(6), 277-290. https://doi.org/10.1016/j.tics.2010.04.004.

Buchsbaum, B. R., \& D'Esposito, M. (2009). Repetition suppression and reactivation in auditory-verbal short-term recognition memory. Cerebral Cortex, 19(6), 1474-1485. https://doi.org/10.1093/ cercor/bhn186.

Buckner, R. L. (2010). Human functional connectivity: New tools, unresolved questions. Proceedings of the National Academy of Sciences of the United States of America, 107(24), 10769-10770. https://doi. org/10.1073/pnas.1005987107.

Chang, C. C., and Lin, C. J. (2011). LIBSVM: A library for support vector machines. Acm Transactions on Intelligent Systems And Technology 2(3). Doi: Artn 27 https://doi.org/10.1145/1961189. 1961199.

Chen, H., Duan, X. J., Liu, F., Lu, F. M., Ma, X. J., Zhang, Y. X., et al. (2016). Multivariate classification of autism spectrum disorder using frequency-specific resting-state functional connectivity-a multi-center study. Progress in Neuro-Psychopharmacology \& Biological Psychiatry, 64, 1-9. https://doi.org/10.1016/j.pnpbp.2015.06.014.

Chen, Y. W., \& Lin, C. J. (2006). Combining SVMs with various feature selection strategies. Feature Extraction, 207, 315-324.

Craddock, R. C., Holtzheimer, P. E., Hu, X. P. P., \& Mayberg, H. S. (2009). Disease state prediction from resting state functional connectivity. Magnetic Resonance in Medicine, 62(6), 1619-1628. https://doi.org/10.1002/mrm.22159.

Davatzikos, C. (2004). Why voxel-based morphometric analysis should be used with great caution when characterizing group differences. Neuroimage, 23(1), 17-20. https://doi.org/10.1016/j.neuroimage. 2004.05.010

Dong, D., Wang, Y., Chang, X., Luo, C., \& Yao, D. (2018). Dysfunction of large-scale brain networks in schizophrenia: A meta-analysis of resting-state functional connectivity. Schizophrenia Bulletin, 44(1), 168-181. https://doi.org/10.1093/schbul/sbx034.

Fox, M. D., \& Raichle, M. E. (2007). Spontaneous fluctuations in brain activity observed with functional magnetic resonance imaging. Nature Reviews. Neuroscience, 8(9), 700-711. https://doi.org/10. 1038/nrn2201.

Fu, C. H. Y., Mourao-Miranda, J., Costafrecla, S. G., Khanna, A., Marquand, A. F., Williams, S. C. R., et al. (2008). Pattern classification of sad facial processing: Toward the development of neurobiological markers in depression. Biological Psychiatry, 63(7), 656662. https://doi.org/10.1016/j.biopsych.2007.08.020.

Golland, P., \& Fischl, B. (2003). Permutation tests for classification: Towards statistical significance in image-based studies. Inf Process Med Imaging, 18, 330-341.

Gong, Q., \& He, Y. (2015). Depression, neuroimaging and connectomics: A selective overview. Biological Psychiatry, 77(3), 223-235. https://doi.org/10.1016/j.biopsych.2014.08.009.

Greicius, M. (2008). Resting-state functional connectivity in neuropsychiatric disorders. Current Opinion in Neurology, 21(4), 424-430. https://doi.org/10.1097/WCO.0b013e328306f2c5.

Greicius, M. D., Flores, B. H., Menon, V., Glover, G. H., Solvason, H. B., Kenna, H., Reiss, A. L., \& Schatzberg, A. F. (2007). Resting-state functional connectivity in major depression: Abnormally increased contributions from subgenual cingulate cortex and thalamus. Biological Psychiatry, 62(5), 429-437. https://doi.org/10.1016/j. biopsych.2006.09.020.

Greicius, M. D., Krasnow, B., Reiss, A. L., \& Menon, V. (2003). Functional connectivity in the resting brain: A network analysis of the default mode hypothesis. Proceedings of the National Academy of Sciences of the United States of America, 100(1), 253-258. https://doi.org/10.1073/pnas.0135058100.

Guo, H., Cheng, C., Cao, X. H., Xiang, J., Chen, J. J., \& Zhang, K. R. (2014a). Resting-state functional connectivity abnormalities in firstonset unmedicated depression. Neural Regeneration Research, 9(2), 153-163. https://doi.org/10.4103/1673-5374.125344.

Guo, W., Liu, F., Yu, M., Zhang, J., Zhang, Z., Liu, J., Xiao, C., \& Zhao, J. (2014b). Functional and anatomical brain deficits in drug-naive major depressive disorder. Progress in Neuro-Psychopharmacology \& Biological Psychiatry, 54, 1-6. https://doi.org/10.1016/j.pnpbp. 2014.05.008.

Guo, W. B., Liu, F., Zhang, J., Zhang, Z. K., Yu, L. Y., Liu, J. R., et al. (2014c). Abnormal default-mode network homogeneity in first-episode, drug-naive major depressive disorder. Plos One 9(3). Doi: ARTN e91102. https://doi.org/10.1371/journal.pone.0091102.

Habas, C., Kamdar, N., Nguyen, D., Prater, K., Beckmann, C. F., Menon, V., \& Greicius, M. D. (2009). Distinct cerebellar contributions to intrinsic connectivity networks. The Journal of Neuroscience, 29(26), 8586-8594. https://doi.org/10.1523/JNEUROSCI.1868-09. 2009.

Hamilton, J. P., Chen, M. C., \& Gotlib, I. H. (2013). Neural systems approaches to understanding major depressive disorder: An intrinsic functional organization perspective. Neurobiology of Disease, 52, 4-11. https://doi.org/10.1016/j.nbd.2012.01.015.

Hamilton, J. P., Farmer, M., Fogelman, P., \& Gotlib, I. H. (2015). Depressive rumination, the default-mode network, and the dark matter of clinical neuroscience. Biological Psychiatry, 78(4), 224-230. https://doi.org/10.1016/j.biopsych.2015.02.020.

Jiang, Y., Duan, M., Chen, X., Chang, X., He, H., Li, Y., et al. (2017). Common and distinct dysfunctional patterns contribute to triple network model in schizophrenia and depression: A preliminary study. Prog Neuropsychopharmacol Biol Psychiatry, 79(Pt B), 302-310. https://doi.org/10.1016/j.pnpbp.2017.07.007.

Kaiser, R. H., Andrews-Hanna, J. R., Wager, T. D., \& Pizzagalli, D. A. (2015). Large-scale network dysfunction in major depressive disorder: A meta-analysis of resting-state functional connectivity. JAMA Psychiatry, 72(6), 603-611. https://doi.org/10.1001/ jamapsychiatry.2015.0071.

Kambeitz, J., Cabral, C., Sacchet, M. D., Gotlib, I. H., Zahn, R., Serpa, M. H., Walter, M., Falkai, P., \& Koutsouleris, N. (2017). Detecting neuroimaging biomarkers for depression: A meta-analysis of multivariate pattern recognition studies. Biological Psychiatry, 82(5), 330-338. https://doi.org/10.1016/j.biopsych.2016.10.028.

Li, J., Duan, X., Cui, Q., Chen, H., \& Liao, W. (2019). More than just statics: Temporal dynamics of intrinsic brain activity predicts the suicidal ideation in depressed patients. Psychological Medicine, 49(5), 852-860. https://doi.org/10.1017/S0033291718001502.

Liao, W., Chen, H., Li, J., Ji, G. J., Wu, G. R., Long, Z., Xu, Q., Duan, X., Cui, Q., \& Biswal, B. B. (2019). Endless fluctuations: Temporal dynamics of the amplitude of low frequency fluctuations. IEEE Transactions on Medical Imaging, 38(11), 2523-2532. https://doi. org/10.1109/TMI.2019.2904555.

Liao, W., Li, J., Duan, X. J., Cui, Q., Chen, H., \& Chen, H. F. (2018). Static and dynamic connectomics differentiate between depressed patients with and without suicidal ideation. Human Brain Mapping, 39(10), 4105-4118. https://doi.org/10.1002/hbm.24235.

Liu, F., Guo, W., Fouche, J. P., Wang, Y., Wang, W., Ding, J., Zeng, L., Qiu, C., Gong, Q., Zhang, W., \& Chen, H. (2015). Multivariate classification of social anxiety disorder using whole brain functional 
connectivity. Brain Structure \& Function, 220(1), 101-115. https:// doi.org/10.1007/s00429-013-0641-4.

Liu, F., Guo, W., Yu, D., Gao, Q., Gao, K., Xue, Z., du, H., Zhang, J., Tan, C., Liu, Z., Zhao, J., \& Chen, H. (2012). Classification of different therapeutic responses of major depressive disorder with multivariate pattern analysis method based on structural MR scans. PLoS One, 7(7), e40968. https://doi.org/10.1371/journal.pone. 0040968.

Liu, R., Yue, Y., Hou, Z., Yuan, Y., \& Wang, Q. (2018). Risk factors associated with cognitions for late-onset depression based on anterior and posterior default mode sub-networks. Journal of Affective Disorders, 235, 544-550. https://doi.org/10.1016/j.jad.2018.04. 065 .

Ma, Q. M., Zeng, L. L., Shen, H., Liu, L., \& Hu, D. W. (2013). Altered cerebellar-cerebral resting-state functional connectivity reliably identifies major depressive disorder. Brain Research, 1495, 86-94. https://doi.org/10.1016/j.brainres.2012.12.002.

Manoliu, A., Meng, C., Brandl, F., Doll, A., Tahmasian, M., Scherr, M., Schwerthöffer D., Zimmer C., Förstl H., Bäuml J., Riedl V., Wohlschläger A. M., Sorg C. (2014a). Insular dysfunction within the salience network is associated with severity of symptoms and aberrant inter-network connectivity in major depressive disorder. Frontiers In Human Neuroscience 7. doi: https://doi.org/10.3389/ fnhum.2013.00930.

Manoliu, A., Riedl, V., Zherdin, A., Muhlau, M., Schwerthoffer, D., Scherr, M., et al. (2014b). Aberrant dependence of default mode/ central executive network interactions on anterior insular salience network activity in schizophrenia. Schizophrenia Bulletin, 40(2), 428-437. https://doi.org/10.1093/schbul/sbt037.

Mantini, D., Perrucci, M. G., Del Gratta, C., Romani, G. L., \& Corbetta, M. (2007). Electrophysiological signatures of resting state networks in the human brain. Proceedings of the National Academy of Sciences of the United States of America, 104(32), 13170-13175. https://doi.org/10.1073/pnas.0700668104.

Meier, T. B., Desphande, A. S., Vergun, S., Nair, V. A., Song, J., Biswal, B. B., Meyerand, M. E., Birn, R. M., \& Prabhakaran, V. (2012). Support vector machine classification and characterization of agerelated reorganization of functional brain networks. Neuroimage, 60(1), 601-613. https://doi.org/10.1016/j.neuroimage.2011.12.052.

Menon, V. (2011). Large-scale brain networks and psychopathology: A unifying triple network model. Trends in Cognitive Sciences, 15(10), 483-506. https://doi.org/10.1016/j.tics.2011.08.003.

Menon, V. (2018). The triple network model, insight, and large-scale brain Organization in Autism. Biological Psychiatry, 84(4), 236238. https://doi.org/10.1016/j.biopsych.2018.06.012.

Mitchell, A. J., Vaze, A., \& Rao, S. (2009). Clinical diagnosis of depression in primary care: A meta-analysis. Lancet, 374(9690), 609-619. https://doi.org/10.1016/S0140-6736(09)60879-5.

Mourao-Miranda, J., Bokde, A. L. W., Born, C., Hampel, H., \& Stetter, M. (2005). Classifying brain states and determining the discriminating activation patterns: Support vector machine on functional MRI data. Neuroimage, 28(4), 980-995. https://doi.org/10.1016/j. neuroimage.2005.06.070.

Mulders, P. C., van Eijndhoven, P. F., Schene, A. H., Beckmann, C. F., \& Tendolkar, I. (2015). Resting-state functional connectivity in major depressive disorder: A review. Neuroscience and Biobehavioral Reviews, 56, 330-344. https://doi.org/10.1016/j.neubiorev.2015. 07.014 .

Nabbe, P., Le Reste, J. Y., Guillou-Landreat, M., Perez, M. A. M., Argyriadou, S., Claveria, A., et al. (2017). Which DSM validated tools for diagnosing depression are usable in primary care research? A systematic literature review. European Psychiatry, 39, 99-105. https://doi.org/10.1016/j.eurpsy.2016.08.004.

Orru, G., Pettersson-Yeo, W., Marquand, A. F., Sartori, G., \& Mechelli, A. (2012). Using support vector machine to identify imaging biomarkers of neurological and psychiatric disease: A critical review.
Neuroscience and Biobehavioral Reviews, 36(4), 1140-1152. https://doi.org/10.1016/j.neubiorev.2012.01.004.

Pereira, F., Mitchell, T., \& Botvinick, M. (2009). Machine learning classifiers and fMRI: A tutorial overview. Neuroimage, 45(1 Suppl), S199-S209. https://doi.org/10.1016/j.neuroimage.2008.11.007.

Power, J. D., Mitra, A., Laumann, T. O., Snyder, A. Z., Schlaggar, B. L., \& Petersen, S. E. (2014). Methods to detect, characterize, and remove motion artifact in resting state fMRI. Neuroimage, 84,320 341. https://doi.org/10.1016/j.neuroimage.2013.08.048.

Radloff, L. S. (1977). A self-report depression scale for research in the general population. Applied Psychological Measurement, 1, 385401.

Raichle, M. E., MacLeod, A. M., Snyder, A. Z., Powers, W. J., Gusnard, D. A., \& Shulman, G. L. (2001). A default mode of brain function. Proceedings of the National Academy of Sciences of the United States of America, 98(2), 676-682. https://doi.org/10.1073/pnas. 98.2.676.

Scholkopf, B., \& Smola, A. J. (2001). Learning with kernels: Support vector machines, regularization, optimization, and beyond. Cambridge: MIT press.

Seeley, W. W., Menon, V., Schatzberg, A. F., Keller, J., Glover, G. H., Kenna, H., Reiss, A. L., \& Greicius, M. D. (2007). Dissociable intrinsic connectivity networks for salience processing and executive control. The Journal of Neuroscience, 27(9), 2349-2356. https://doi.org/10.1523/JNEUROSCI.5587-06.2007.

Sexton, C. E., Allan, C. L., Le Masurier, M., McDermott, L. M., Kalu, U. G., Herrmann, L. L., et al. (2012). Magnetic resonance imaging in late-life depression: Multimodal examination of network disruption. Archives of General Psychiatry, 69(7), 680-689. https://doi.org/10. 1001/archgenpsychiatry.2011.1862.

Silani, G., Lamm, C., Ruff, C. C., \& Singer, T. (2013). Right Supramarginal Gyrus is crucial to overcome emotional egocentricity Bias in social judgments. Journal of Neuroscience, 33(39), 1546615476. https://doi.org/10.1523/Jneurosci.1488-13.2013.

Smith, K. (2012). Brain imaging: fMRI 2.0. Nature, 484(7392), 24-26. https://doi.org/10.1038/484024a.

Supekar, K., Cai, W., Krishnadas, R., Palaniyappan, L., \& Menon, V. (2019). Dysregulated brain dynamics in a triple-network saliency model of schizophrenia and its relation to psychosis. Biological Psychiatry, 85(1), 60-69. https://doi.org/10.1016/j.biopsych.2018. 07.020 .

Tian, L., Wang, J., Yan, C., \& He, Y. (2011). Hemisphere- and genderrelated differences in small-world brain networks: A resting-state functional MRI study. Neuroimage, 54(1), 191-202. https://doi. org/10.1016/j.neuroimage.2010.07.066.

Tzourio-Mazoyer, N., Landeau, B., Papathanassiou, D., Crivello, F., Etard, O., Delcroix, N., Mazoyer, B., \& Joliot, M. (2002). Automated anatomical labeling of activations in SPM using a macroscopic anatomical parcellation of the MNI MRI single-subject brain. Neuroimage, 15(1), 273-289. https://doi.org/10.1006/nimg. 2001.0978

Vapnik, V. (2000). The nature of statistical learning theory. Springer.

Wang, C. J. (2007). Credit scoring with a data mining approach based on support vector machines. Expert Systems with Applications, 33(4), $847-856$.

Wang, L. B., Shen, H., Tang, F., Zang, Y. F., \& Hu, D. W. (2012). Combined structural and resting-state functional MRI analysis of sexual dimorphism in the young adult human brain: An MVPA approach. Neuroimage, 61(4), 931-940. https://doi.org/10.1016/j. neuroimage.2012.03.080.

Wang, M., Armour, C., Wu, Y., Ren, F., Zhu, X., \& Yao, S. (2013). Factor structure of the CES-D and measurement invariance across gender in mainland Chinese adolescents. Journal of Clinical Psychology, 69(9), 966-979. https://doi.org/10.1002/jclp.21978.

Wolfers, T., Buitelaar, J. K., Beckmann, C. F., Franke, B., \& Marquand, A. F. (2015). From estimating activation locality to predicting 
disorder: A review of pattern recognition for neuroimaging-based psychiatric diagnostics. Neuroscience and Biobehavioral Reviews, 57, 328-349. https://doi.org/10.1016/j.neubiorev.2015.08.001.

World Health Organization. (2017). Depression and other common mental disorders Global Health estimates. Geneva: World Health Organization.

Yan, C. G., Cheung, B., Kelly, C., Colcombe, S., Craddock, R. C., Di Martino, A., et al. (2013). A comprehensive assessment of regional variation in the impact of head micromovements on functional connectomics. Neuroimage, 76, 183-201. https://doi.org/10.1016/ j.neuroimage.2013.03.004

Zeng, L. L., Shen, H., Liu, L., Wang, L. B., Li, B. J., Fang, P., et al. (2012). Identifying major depression using whole-brain functional connectivity: A multivariate pattern analysis. Brain, 135, 14981507. https://doi.org/10.1093/brain/aws059.

Zheng, H., Xu, L., Xie, F., Guo, X., Zhang, J., Yao, L., \& Wu, X. (2015). The altered triple networks interaction in depression under resting state based on graph theory. BioMed Research International, 2015, 386326-386328. https://doi.org/10.1155/2015/386326.

Zheng, Y., Chen, X., Li, D., Liu, Y., Tan, X., Liang, Y., Zhang, H., Qiu, S., \& Shen, D. (2019). Treatment-naive first episode depression classification based on high-order brain functional network. Journal of Affective Disorders, 256, 33-41. https://doi.org/10. 1016/j.jad.2019.05.067.
Zhong, X., Shi, H. Q., Ming, Q. S., Dong, D. F., Zhang, X. C., Zeng, L. L., et al. (2017). Whole-brain resting-state functional connectivity identified major depressive disorder: A multivariate pattern analysis in two independent samples. Journal of Affective Disorders, 218, 346-352. https://doi.org/10.1016/j.jad.2017.04.040.

Zhu, C. Z., Zang, Y. F., Cao, Q. J., Yan, C. G., He, Y., Jiang, T. Z., Sui, M. Q., \& Wang, Y. F. (2008). Fisher discriminative analysis of resting-state brain function for attention-deficit/hyperactivity disorder. Neuroimage, 40(1), 110-120. https://doi.org/10.1016/j. neuroimage.2007.11.029.

Zhu, X., Wang, X., Xiao, J., Liao, J., Zhong, M., Wang, W., \& Yao, S. (2012). Evidence of a dissociation pattern in resting-state default mode network connectivity in first-episode, treatment-naive major depression patients. Biological Psychiatry, 71(7), 611-617. https:// doi.org/10.1016/j.biopsych.2011.10.035.

Zhu, X., Zhu, Q., Shen, H., Liao, W., \& Yuan, F. (2017). Rumination and default mode network subsystems connectivity in first-episode, drug-naive young patients with major depressive disorder. Scientific Reports, 7, 43105. https://doi.org/10.1038/srep43105.

Publisher's note Springer Nature remains neutral with regard to jurisdictional claims in published maps and institutional affiliations. 